This item was submitted to Loughborough's Institutional Repository (https://dspace.lboro.ac.uk/) by the author and is made available under the following Creative Commons Licence conditions.

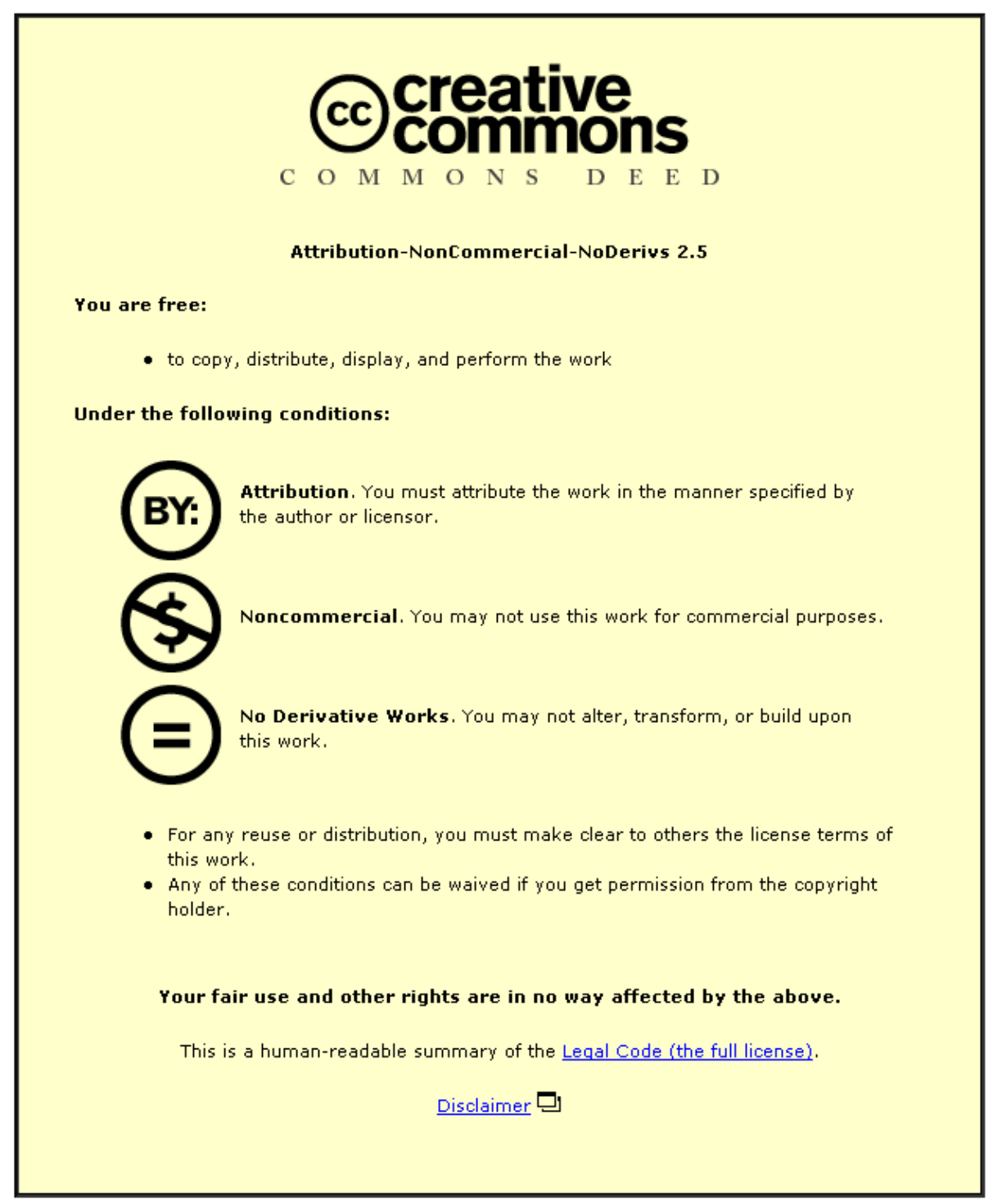

For the full text of this licence, please go to: http://creativecommons.org/licenses/by-nc-nd/2.5/ 


\title{
Evolution of the Light Emission Profile in Radio-Frequency Atmospheric Pressure Glow Discharges
}

\author{
Dawei Liu, Felipe Iza, Member, IEEE, and Michael G. Kong, Senior Member, IEEE
}

\begin{abstract}
Time-resolved images of the light emission profiles of RF atmospheric pressure plasmas are presented. As the current increases, the emission profile evolves from bell shaped to double humped. At moderate currents, bright layers above each of the electrodes are observed, and it is found that both layers light up and fade simultaneously during the RF cycle.
\end{abstract}

Index Terms-Atmospheric plasma, CCD imaging, glow discharge, RF discharge.

A TMOSPHERIC pressure glow discharges (APGDs) have received growing attention in recent years for their potential application in many scientific disciplines [1], [2]. Despite recent advances, the physics governing these discharges is not fully understood. In this paper, the dynamic evolution of the optical emission of $\mathrm{He}$ and $\mathrm{He}+\mathrm{O}_{2} \mathrm{RF}$ (sinusoid $13.56 \mathrm{MHz}$ ) APGDs is investigated by means of high-speed (5-ns exposure time) images.

The experimental setup consists of two parallel stainlesssteel disk electrodes, each being $2 \mathrm{~cm}$ in diameter. The discharge gap is fixed at $2 \mathrm{~mm}$, and the system is housed in a Perspex box. Working gases ( $\mathrm{He}$ and $\mathrm{He}+\mathrm{O}_{2}$ mixtures) are flowed at $5 \mathrm{slm}$ into the box. A function generator (Tektronix AFG 3102), an RF power amplifier (Amplifier Research 500A100A), and a house-built matching network are used to deliver power to the discharge. The current flowing through the discharge and the voltage across the electrodes are measured with a wideband current probe (Pearson Current Monitor 2877), a wideband voltage probe (Tektronix P5100), and a digital oscilloscope (Tektronix TDS 5054B). An intensified charge-coupled device camera (Andor i-Star DH720) is used to take the images presented in this paper [3]. The electron temperature and density were not measured in this set of experiments. The gas temperature (estimated from the rotational temperature of $\mathrm{OH}$ ) increases from $310 \mathrm{~K}$ at $60 \mathrm{~mA}$ to $430 \mathrm{~K}$ at $180 \mathrm{~mA}$ in $\mathrm{He}$ and from $340 \mathrm{~K}$ at $90 \mathrm{~mA}$ to $470 \mathrm{~K}$ at $208 \mathrm{~mA}$ in $\mathrm{He}+\mathrm{O}_{2}$.

Fig. 1 shows the current-voltage characteristics of the He and $\mathrm{He}+\mathrm{O}_{2}(0.5 \%)$ discharges from (dotted lines) prebreakdown

Manuscript received November 5, 2007; revised January 15, 2008. This work was supported by the Engineering and Physical Science Research Council (U.K.).

The authors are with the Department of Electronic and Electrical Engineering, Loughborough University, LE11 3TU Leicestershire, U.K. (e-mail: f.iza@lboro.ac.uk).

Digital Object Identifier 10.1109/TPS.2008.922426

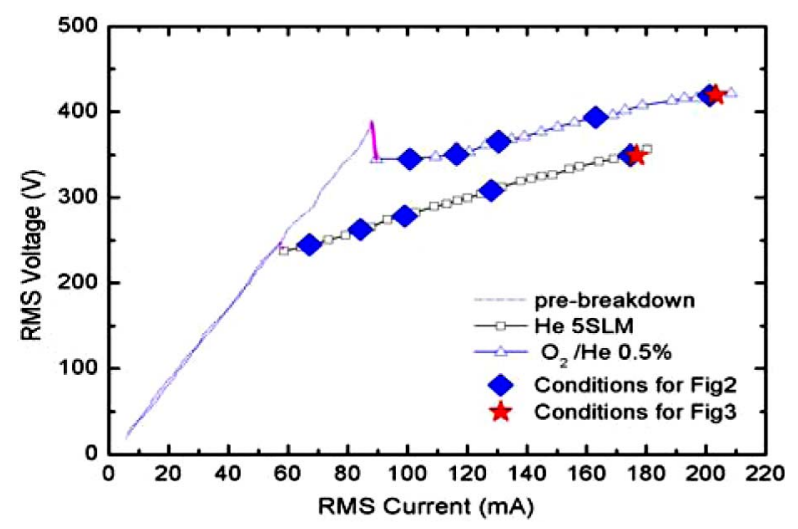

Fig. 1. Current-voltage curve of RF APGDs in $\mathrm{He}$ and $\mathrm{He}+\mathrm{O}_{2}$.

to (open symbols) glow discharge and up to the (red stars) point immediately before the plasma constriction. In both cases, right after the discharge ignites, the voltage required to sustain the plasma decreases (solid pink lines in Fig. 1). Due to the high electron affinity of $\mathrm{O}_{2}$ and its molecular nature, a higher voltage is required to ignite the discharge even when only $0.5 \%$ of $\mathrm{O}_{2}$ is added into the flow.

The time-integrated light emission profile of the discharge as the current (or power) increases is shown in Fig. 2. Each image was captured by using an exposure time of $500 \mathrm{~ns}(\sim 7 \mathrm{RF}$ cycles), and the corresponding points in the current-voltage characteristic are highlighted by blue diamonds in Fig. 1. Immediately after breakdown, the plasma is weak and covers the electrodes only partially. As current is increased, the plasma expands until it covers the entire electrodes. The light emission profile evolves from bell-like to double-hump profile as the current increases. The latter presents two distinct bright emission layers above each of the electrodes (Fig. 2). This structure is also observed when $\mathrm{O}_{2}$ is added into the discharge, although the overall intensity of the discharge decreases significantly. It is of interest to note that the decrease of light emission with $\mathrm{O}_{2}$ admixtures happens even though higher power is delivered to the plasma.

To resolve the time evolution of the plasma emission, singleshot images with an exposure time of $5 \mathrm{~ns}$ were taken at different phases of the RF cycle (Fig. 3). Both discharges (He and $\mathrm{He}+\mathrm{O}_{2}$ ) operate at the maximum current before constriction (red stars in Fig. 1). As it was observed in previous works [4], the emission is periodic, and the maximum light is recorded 


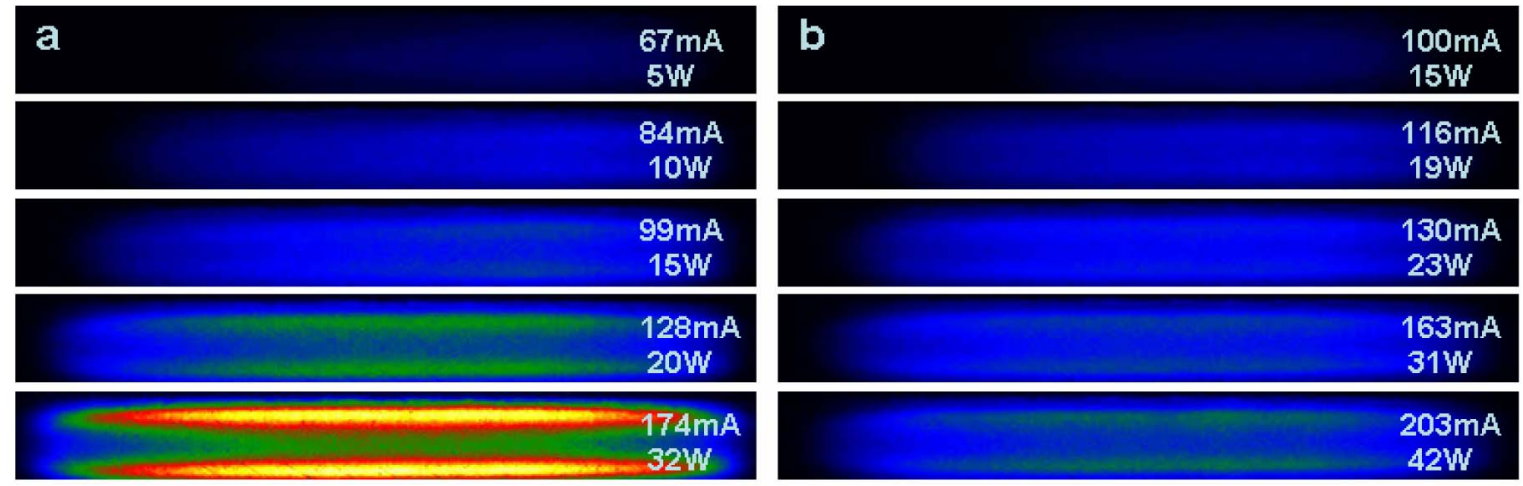

Fig. 2. Emission evolution as a function of the discharge current. Time-integrated optical emission of (a) $\mathrm{He}$ and (b) $\mathrm{He}+\mathrm{O}_{2} 0.5 \%$ plasmas.
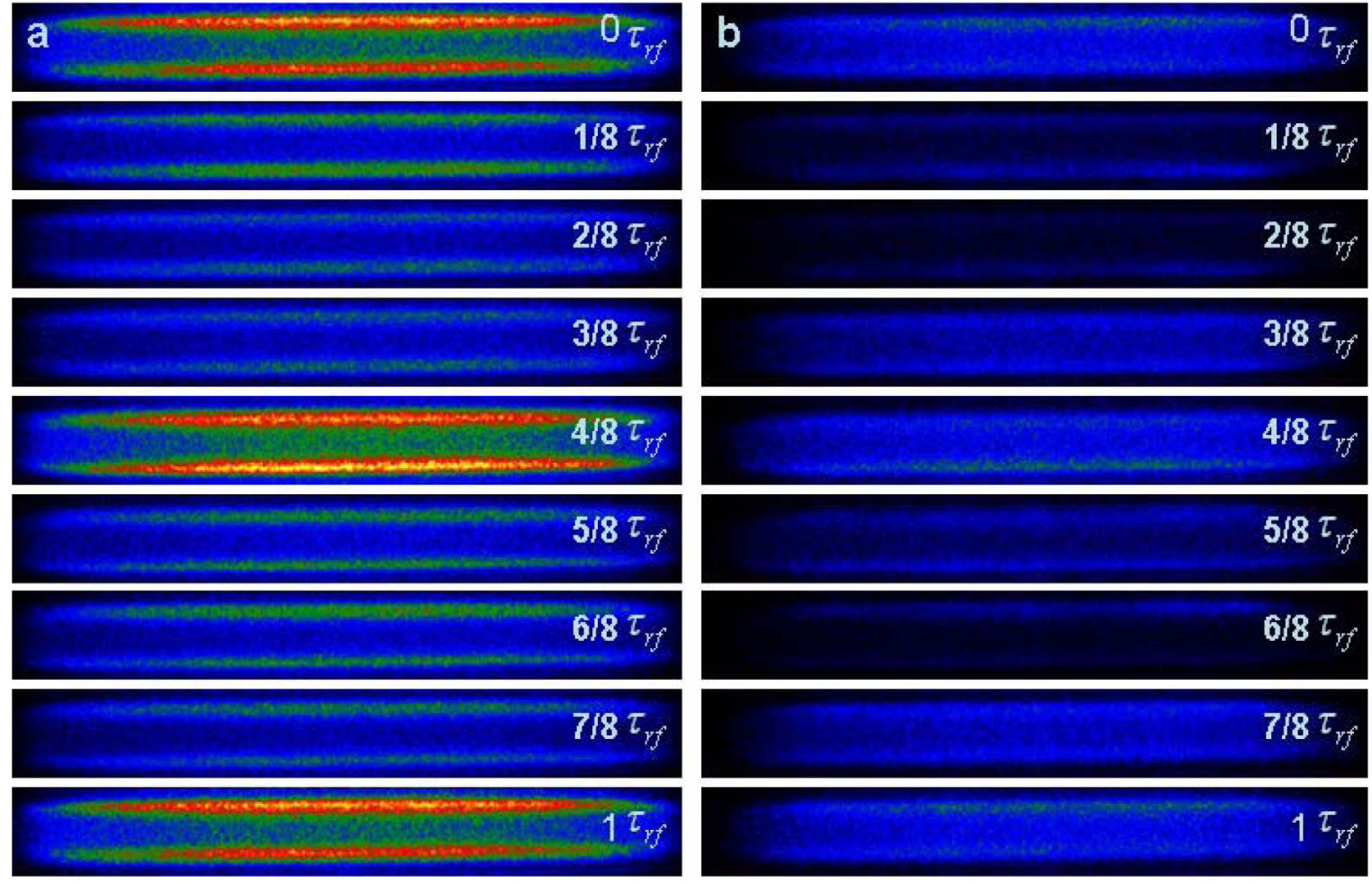

Fig. 3. Single-shot images (5-ns exposure time) over one complete RF cycle. (a) He discharge at $174 \mathrm{~mA}$ and (b) $\mathrm{He}+\mathrm{O}_{2}$ discharge at $203 \mathrm{~mA}$.

with a period equal to half the RF period $\left(\tau_{\mathrm{RF}}\right)$. It is interesting to note that the two bright layers above each electrode light up simultaneously and not in an alternating fashion. Although bright emission layers near the electrodes are often associated with the gamma mode, the simultaneous lightening of the layers and the lack of discharge constriction are signatures of operation in the alpha mode. Simulation results not presented here also support this conclusion. In the presence of $\mathrm{O}_{2}$, the same observations hold although the intensity of the bright layers is less prominent.

In summary, the $\mathrm{He}$ and $\mathrm{He}+\mathrm{O}_{2}$ RF APGDs have been studied by means of single-shot images with 5 -ns exposure time. As a rule, the presence of $\mathrm{O}_{2}$ decreases the light emission even if more power is delivered to the discharge, and the emission profile evolves from bell-like to double-humped profile as the current increases. In all cases, the discharge operates in the alpha mode, and the bright layers that are observed above each electrode light up and fade simultaneously (not alternatively) with a period of $\tau_{\mathrm{RF}} / 2$.

\section{REFERENCES}

[1] J. R. Roth, "Potential industrial applications of the one atmosphere uniform glow discharge plasma operating in ambient air," Phys. Plasmas, vol. 12, no. 5, p. 057 103, May 2005.

[2] J. G. Eden, "Information display early in the 21 st century: Overview of selected emissive display technologies," Proc. IEEE, vol. 94, no. 3, pp. 567574, Mar. 2006.

[3] J. L. Walsh, J. J. Shi, and M. G. Kong, "Submicrosecond pulsed atmospheric glow discharge sustained without dielectric barriers at kilohertz frequencies," Appl. Phys. Lett., vol. 89, no. 16, p. 161505 , Oct. 2006

[4] D. W. Liu, J. J. Shi, and M. G. Kong, "Electron trapping in radio-frequency atmospheric-pressure glow discharges," Appl. Phys. Lett., vol. 90, no. 4, p. 041502 , Jan. 2007. 